\title{
ESTAÇÃO CHUVOSA E DE CULTIVO PARA A REGIÃO DE RIO LARGO, ALAGOAS BASEADA EM MÉTODOS DIRETOS E SUA RELAÇÃO COM O EL NIÑO - OSCILAÇÃO SUL
}

\author{
ANDRÉ LUIZ DE CARVALHO ${ }^{1}$, JOSÉ LEONALDO DE SOUZA², GUSTAVO BASTOS LYRA ${ }^{3}$, \\ EDUARDO CABRAL DA SILVA ${ }^{4}$
}

\author{
${ }^{1}$ Universidade Federal de Pernambuco (UFPE), Departamento de Energia Nuclear, Recife, PE, Brasil \\ ${ }^{2}$ Universidade Federal de Alagoas (UFAL), Instituto de Ciências Atmosféricas, Maceió, AL, Brasil \\ ${ }^{3}$ Universidade Federal Rural do Rio de Janeiro (UFRRJ), Instituto de Florestas, Rio de Janeiro, RJ, Brasil \\ ${ }^{4}$ UFPE, Centro de Tecnologia e Geociências, Recife, PE, Brasil
}

del.andre2@hotmail.com, leonaldojs@yahoo.com.br, gblyra@ufrrj.br, edcs_cabral@hotmail.com

Recebido Março de 2012 - Aceito Outubro de 2012

\begin{abstract}
RESUMO
A precipitação pluvial é um dos principais fatores meteorológicos responsáveis pelo rendimento das culturas agrícolas. A sua irregularidade, observada de forma mais intensa na região Nordeste, causa a alternância das produções agrícolas anuais. Assim, esse trabalho objetivou determinar as características da estação chuvosa e de cultivo (início, fim e comprimento) baseado em dados diários de precipitação pluvial, entre 1973 e 2008, para o município de Rio Largo, Alagoas. O início (potencial e de sucesso), fim e comprimento da estação chuvosa e de cultivo foram definidos pela utilização de métodos diretos em função da precipitação pluvial e evapotranspiração de referência. As análises estatísticas das características da estação chuvosa e de cultivo foram realizadas pelo aplicativo Instat Climatic. A estação chuvosa, esperada a $80 \%$ de probabilidade, iniciou-se em 07 de abril e terminou em 24 de outubro, com comprimento de 221 dias. O início da estação chuvosa foi antecipado em anos de La Niña (dez dias) e retardado em alguns anos de El Niño (nove dias), e assim, ocorre diminuição no comprimento da estação de cultivo para anos de El Niño e aumento em anos de La Niña.
\end{abstract}

Palavras-chave: precipitação pluvial; estação chuvosa; ENOS.

\section{ABSTRACT: CULTIVATION AND RAINY SEASON IN THE REGION OF RIO LARGO, ALAGOAS BASED ON DIRECT METHODS AND ITS RELATION WITH EL NIÑO - SOUTH OSCILATION.}

Rainfall is one of the main meteorological factors responsible for agricultural crops yield. Its irregularity observed more intensely in the Northeast causes the alternation of annual agricultural production. Thus, this study aimed to determine the characteristics of the wet and growing season based on daily rainfall data between 1973 and 2008 in Rio Largo, Alagoas. The beginning (potencial and success), ending and length of the cultivation and rainy season were determinate by direct methods using daily rainfall and reference evapotranspiration. Statistical analysis of cultivation and rainy season characteristics were done using the Instat Climatic software. The expected rainy season, at $80 \%$ probability, began on April $7^{\text {th }}$ and finished on October $24^{\text {th }}$, with a duration of 221 days. The beginning of the rainy season was anticipated in La Niña years (ten days) and postponed in years of El Niño (nine days) and consequently, cause a decrease in the cultivation length season for El Niño years and increase in La Niña years.

Keywords: rainfall; rainy season; ENSO 


\section{INTRODUÇÃO}

As culturas agrícolas necessitam, para seu adequado crescimento e desenvolvimento, de determinada quantidade de água, que é suprida, principalmente, pela precipitação pluvial, e em parte pela irrigação. A precipitação pluvial está diretamente relacionada com o rendimento final das culturas e sua variação durante $\mathrm{o}$ ano. Porém, associado a outros fatores, pode provocar perdas na produtividade final das culturas (Morais et al., 2001; Mousinho et al., 2006). Na região Nordeste os principais sistemas responsáveis pela ocorrência de precipitação pluvial são: a Zona de Convergência Intertropical (ZCIT), Zona de Convergência do leste do Nordeste (ZCEN), Brisas (marítima e terrestre), as Perturbações Ondulatórias nos ventos Alísios (POAS) e os Vórtices Ciclônicos de Altos Níveis (VCANs) (Molion e Bernardo, 2002; Ferreira e Melo, 2005).

Além da distribuição irregular da chuva, no tempo e no espaço, outra característica climática que afeta essa região é a transição entre períodos secos e chuvosos. Essa transição provoca notável mudança no clima, principalmente, em regiões tropicais (Keller Filho et al., 2006). A variabilidade interanual e intrasazonal da precipitação pluvial no Nordeste também é influenciada por mecanismos de escala global como eventos de ENOS - El Niño Oscilação Sul (Da Silva, 2005). O ENOS é um fenômeno atmosférico de interação oceano-atmosfera, que ocorre no oceano Pacífico Tropical, e é considerado como a principal causa da variabilidade climática em diversas regiões do globo. Ele apresenta duas fases extremas: uma fase quente, denominada El Niño, e uma fase fria, a La Niña (Andreoli e Kayano, 2005; Molion, 2005).

Como as culturas necessitam de elevada quantidade de água, a melhor época para realizar a semeadura é a estação chuvosa (Azevedo e Maciel, 1993). Uma forma de atenuar a falta de água nas culturas é utilizar a data de início de chuvas como a data de plantio (Sivakumar, 1988), mas, o plantio também pode ser realizado durante a estação seca, sendo necessária a utilização da irrigação para suprir a quantidade de água para as culturas.

O objetivo desse trabalho foi determinar as características da estação chuvosa e de cultivo baseado em dados diários de precipitação pluvial para o município de Rio Largo, Alagoas.

\section{MATERIAL E MÉTODOS}

O estudo foi realizado com base em dados diários de precipitação, observados entre 1973 e 2008 na região de Rio Largo $\left(9^{\circ} 28^{\prime} \mathrm{S}, 35^{\circ} 49^{\prime} \mathrm{W}, 127 \mathrm{~m}\right)$. A precipitação foi obtida em pluviômetro convencional instalado na estação agrometeorológica no Centro de Ciências Agrárias (CECA) da Universidade Federal de Alagoas (UFAL). Rio Largo é localizado na Região Metropolitana de Maceió - tabuleiros costeiros de Alagoas. O clima dessa região, segundo a classificação climática de Köppen, é tropical litorâneo úmido (As), com baixa amplitude térmica anual e com maiores totais de precipitação entre abril e julho (Cardim, 2003). Os valores totais anuais de precipitação pluvial são em média $1.800 \mathrm{~mm}$. A temperatura do ar, a $80 \%$ de probabilidade, apresenta variação de 26,0 e $32,8^{\circ} \mathrm{C}$, para a temperatura máxima, e de $18,3^{\circ} \mathrm{C}$ e $23,2^{\circ} \mathrm{C}$, para a temperatura mínima (Souza et al., 2004).

O tratamento dos dados e as análises estatísticas foram realizados com o auxílio do aplicativo Instat Climatic, que é uma ferramenta para análises estatísticas de eventos climáticos (Stern et al., 2005). O método direto (distribuição de frequência) para identificação do início potencial da estação chuvosa e de cultivo, consistiu na determinação do primeiro dia de março, que é o mês de transição entre o período seco e o período úmido (Souza et al., 2004), com precipitação de $20 \mathrm{~mm}$ ou superior, em um ou dois dias consecutivos (Stern et al., 1982). Já o início de sucesso consistiu no mesmo critério do início potencial, mas sem a ocorrência de períodos secos (períodos com ocorrência de dias secos consecutivos) igual ou maior a 10 dias nos 30 dias posteriores. O fim da estação chuvosa com método direto consistiu no primeiro dia depois do dia primeiro de setembro (mês de início do período seco), quando a máxima capacidade de armazenamento de água do solo $(60 \mathrm{~mm})$ diminui até zero, contabilizando-se a precipitação pluvial e a evapotranspiração de referência diária. O comprimento em número de dias da estação de cultivo foi determinado pela diferença entre o início e o fim das chuvas. A evapotranspiração de referência diária $\left(\mathrm{ET}_{0}, \mathrm{~mm} \mathrm{~d}^{-1}\right)$ foi determinada pelo modelo de Penman-Monteith parametrizado no boletim FAO56 (Allen et al., 1998):

$$
E T_{O}=\frac{0,408 \Delta\left(R_{n}-G\right)+\gamma \frac{900}{T_{a}+273} u_{2}\left(e_{s}-e_{a}\right)}{\Delta+\gamma\left(1+0,34 u_{2}\right)}
$$

em que, $\mathrm{T}_{\mathrm{a}}\left({ }^{\circ} \mathrm{C}\right)$ é a temperatura média diária do ar a $2 \mathrm{~m}$ de altura $\left[\mathrm{T}_{\mathrm{a}}=\left(\mathrm{T}_{\mathrm{x}}+\mathrm{T}_{\mathrm{n}}\right) / 2\right] ; \mathrm{T}_{\mathrm{x}}$ e $\mathrm{T}_{\mathrm{n}}\left({ }^{\circ} \mathrm{C}\right)$ são as temperaturas do ar máxima e mínima diária, respectivamente; $\Delta\left(\mathrm{kPa}^{\circ} \mathrm{C}^{-1}\right)$ é a derivada da curva de pressão de saturação do vapor d'água do ar no ponto de $\mathrm{T}_{\mathrm{a}} ; \mathrm{Rn}\left(\mathrm{MJ} \mathrm{m}^{-2} \mathrm{~d}^{-1}\right)$ é o saldo de radiação total diário; $\mathrm{G}\left(\mathrm{MJ} \mathrm{m}^{-2} \mathrm{~d}^{-1}\right)$ é o fluxo de calor no solo; $\gamma\left(\mathrm{kPa}^{\circ} \mathrm{C}^{-1}\right)$ é o coeficiente psicrométrico; es e ea $(\mathrm{kPa})$ são as médias diárias das pressões de saturação e parcial de vapor d'água do ar, respectivamente, e $\mathrm{u}_{2}\left(\mathrm{~m} \mathrm{~s}^{-1}\right)$ é a média diária da velocidade do vento a $2 \mathrm{~m}$ de altura. As medidas dos elementos meteorológicos utilizados para estimativa de $\mathrm{ET}_{\mathrm{o}}$ foram realizadas na estação agrometeorológica do CECA.

As datas de início, fim e o comprimento da estação chuvosa e de cultivo foram ajustados a uma distribuição normal para comparar os resultados com a distribuição observada: 


$$
f(x)=\frac{1}{\sqrt{2 \pi \sigma}} e^{-\frac{1}{2}\left(\frac{x-\mu}{\sigma}\right)^{2}}
$$

em que, $\mu$ é a média e $\sigma$ o desvio padrão da população.

A análise das características da estação chuvosa, com os eventos de ENOS - El Niño Oscilação Sul, foi realizada pela classificação dos anos; em anos de ocorrência de eventos de El Niño e anos de ocorrência de eventos de La Niña, para a série de dados de estudo, disponibilizada pelo Centro de Previsão de Tempo e Estudos Climáticos / Instituto Nacional de Pesquisas Espaciais (CPTEC/INPE) no site: http://enos.cptec.inpe.br/ tab_elnino.shtml e http://enos.cptec.inpe.br/tab_lanina.shtml (Tabela 1).

\section{RESULTADOS E DISCUSSÕES}

A distribuição de frequência observada para o início da estação chuvosa e de cultivo mostrou que o início potencial ocorreu, a $20 \%$ de probabilidade, em $03 / 03$ e a $80 \%$ em $01 / 04$ (Figura 1). O nível de $80 \%$ de probabilidade é recomendado em climatologia agrícola para garantir que a cada dez anos ocorram oito chance de ser observado um evento igual ou

Tabela 1 - Classificação e Intensidade do El Niño - Oscilação Sul no período de 1973 a 2008.

\begin{tabular}{ccc}
\hline Período & Classificação & Intensidade \\
\hline $1972-1973$ & El Niño & Forte \\
$1973-1976$ & La Niña & Forte \\
$1976-1977$ & El Niño & Fraco \\
$1977-1978$ & El Niño & Fraco \\
$1979-1980$ & El Niño & Fraco \\
$1982-1983$ & El Niño & Forte \\
$1983-1984$ & La Niña & Fraco \\
$1984-1985$ & La Niña & Fraco \\
$1986-1988$ & El Niño & Moderado \\
$1988-1989$ & La Niña & Forte \\
$1990-1993$ & El Niño & Forte \\
$1994-1995$ & El Niño & Moderado \\
$1995-1996$ & La Niña & Fraco \\
$1997-1998$ & El Niño & Forte \\
$1998-2001$ & La Niña & Moderado \\
$2002-2003$ & El Niño & Moderado \\
$2004-2007$ & El Niño & Fraco \\
2008 & La Niña & Forte \\
\hline
\end{tabular}

Fonte: CPTEC/INPE

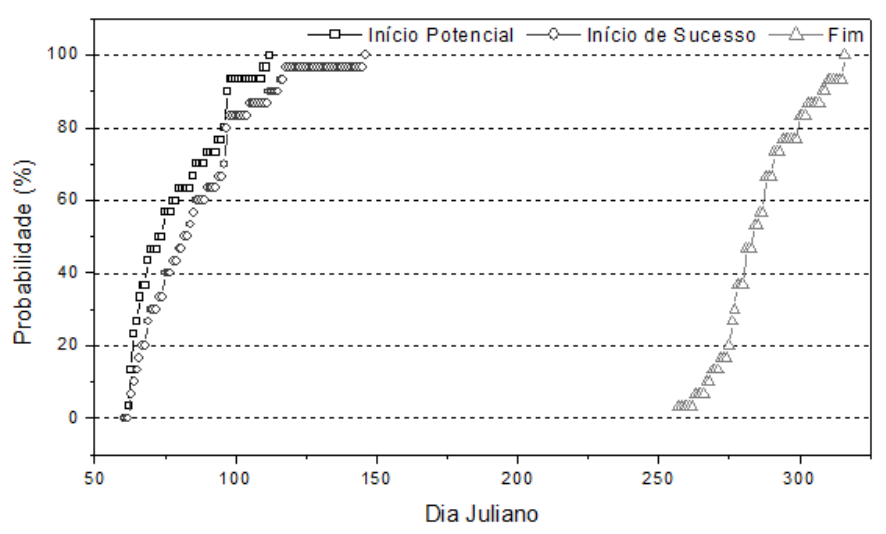

Figura 1 - Probabilidade Cumulativa do Início Potencial, Início de Sucesso e Fim da estação chuvosa no período de 1973 - 2008, Rio Largo, AL.

superior. O início de sucesso (05/03), a 20\% de probabilidade, diferiu do início potencial (03/03) por apenas dois dias (Tabela 2 ), enquanto a $80 \%$ a diferença foi de seis dias $(07 / 04)$. A diferença entre os inícios potencial e de sucesso da estação chuvosa e de cultivo foi de aproximadamente 30 dias entre as probabilidades 20 e $80 \%$. O fim da estação chuvosa e de cultivo teve maior chance de ocorrência em outubro. Em $20 \%$ dos anos, o fim ocorreu em $01 / 10$, enquanto que a $80 \%$ de probabilidade foi em 24/10 (29 anos). Isso foi equivalente, para 24/10, o armazenamento de $60 \mathrm{~mm}$ ser totalmente utilizado no processo de evapotranspiração da região. A análise da probabilidade de ocorrência do início da estação chuvosa foi descrita por Stern et al., (1982) em um estudo realizado em Kano na Nigéria. Nessa região o início potencial e o início de sucesso ocorreram, a $80 \%$ de probabilidade, em 16 e 28 de junho, respectivamente. O fim da estação chuvosa ocorreu, ao mesmo nível de probabilidade, em 28 de setembro.

A proximidade nos valores do início potencial e do início de sucesso da estação chuvosa e de cultivo resultou em comprimentos similares desses períodos (Tabela 3), visto que o método de determinação do fim foi o mesmo em ambos os casos. O comprimento da estação chuvosa e de cultivo para $20 \%$ de probabilidade foi de 197 e de 187 dias para o início potencial e de sucesso, respectivamente. Ou seja, para $20 \%$ dos anos (sete anos) a diferença foi de dez dias. Esses períodos de cultivo a $80 \%$ de probabilidade tiveram 225 e 221 dias para o início potencial e de sucesso, o que correspondeu à diferença de quatro dias em 29 anos da série de 1973 a 2008. O comprimento médio do período de cultivo foi de 208 dias (início potencial) e 203 dias (início de sucesso), com a diferença de apenas cinco dias entre os métodos. Em Kano, o comprimento médio foi de 124 dias (Stern et al., 1982).

A caracterização da estação chuvosa de Rio Largo foi realizada por Cardim (2003), baseada no método da precipitação 
Tabela 2 - Característica da estação chuvosa e de cultivo baseada na distribuição livre e ajustada pela distribuição normal para a região de Rio Largo, AL.

\begin{tabular}{ccccccc}
\hline \multirow{2}{*}{$\begin{array}{c}\text { Porcentagem } \\
(\%)\end{array}$} & $\begin{array}{c}\text { Início } \\
\text { potencial }\end{array}$ & $\begin{array}{c}\text { Início } \\
\text { Sucesso }\end{array}$ & Fim & $\begin{array}{c}\text { Início } \\
\text { potencial }\end{array}$ & $\begin{array}{c}\text { Início } \\
\text { Sucesso }\end{array}$ & Fim \\
& $03 / 03$ & $03 / 03$ & $24 / 09$ & $27 / 02$ & $28 / 02$ & $23 / 09$ \\
10 & $03 / 03$ & $05 / 03$ & $01 / 10$ & $04 / 03$ & $07 / 03$ & $30 / 09$ \\
20 & $13 / 03$ & $18 / 03$ & $10 / 10$ & $16 / 03$ & $23 / 03$ & $11 / 10$ \\
50 & $01 / 04$ & $07 / 04$ & $24 / 10$ & $29 / 03$ & $09 / 04$ & $23 / 10$ \\
80 & $06 / 04$ & $22 / 04$ & $04 / 11$ & $04 / 04$ & $17 / 04$ & $29 / 10$ \\
90 & & & & & & \\
\hline
\end{tabular}

Tabela 3 - Comprimento da estação chuvosa e de cultivo baseado na distribuição livre e na ajustada pela distribuição normal, para a região de Rio Largo, AL.

\begin{tabular}{ccccc}
\hline $\begin{array}{c}\text { Porcentagem } \\
(\%)\end{array}$ & $\begin{array}{c}\text { Distribuição Observada } \\
\text { Comprimento } \\
\text { (Fim - Iníc.Pot.) }\end{array}$ & $\begin{array}{c}\text { Comprimento } \\
\text { (Fim -Iníc.Suc.) }\end{array}$ & $\begin{array}{c}\text { Domprimento } \\
\text { (Fim - Iníc. Pot.) }\end{array}$ & $\begin{array}{c}\text { Comprimento } \\
\text { (Fim - Iníc.Suc.) }\end{array}$ \\
\hline 10 & 185 & 174 & 185 & 171 \\
20 & 197 & 187 & 193 & 182 \\
50 & 208 & 203 & 209 & 202 \\
80 & 225 & 221 & 225 & 222 \\
90 & 232 & 232 & 233 & 233 \\
\hline
\end{tabular}

provável de 20 mm e no método da precipitação provável a 75\% de probabilidade e a evapotranspiração, em períodos decendiais. Esses métodos mostraram que o início da estação chuvosa ocorreu no decêndio entre os dias 01-10/04. Esses resultados mostram que métodos diretos são úteis para a determinação do início da estação chuvosa, pois a $80 \%$ de probabilidade, o início potencial ocorreu em 01/04 e o início de sucesso ocorreu em 07/04. O fim e o comprimento da estação chuvosa apresentaram valores diferentes para os métodos avaliados por Cardim (2003). No método de precipitação provável a $20 \mathrm{~mm}$ e a $75 \%$ de probabilidade, o fim da estação chuvosa ocorreu no decêndio entre 01-10/10. Mas, o método direto mostrou que o fim da estação chuvosa, a $80 \%$ de probabilidade, ocorreu no fim de outubro (24/10). O comprimento da estação, que foi de 193 dias para os métodos de precipitação provável a $20 \mathrm{~mm}$ e a $75 \%$, para o método direto foi de 225 dias, considerando o início potencial e 221 dias dado o início de sucesso

Baseado na distribuição normal ajustada (Tabela 2), o início potencial e de sucesso da estação chuvosa e de cultivo também apresentaram proximidade. A $20 \%$ de probabilidade, o início potencial da estação chuvosa e de cultivo foi observado em $04 / 03$, enquanto que o início de sucesso foi para $07 / 03$, ou seja, a diferença foi de apenas três dias. A $80 \%$ de probabilidade, o início potencial da estação chuvosa e de cultivo ocorreu em 29/03 e o início de sucesso foi em $09 / 04$, e assim, diferença de 11 dias.

A distribuição normal, quando comparada com a distribuição de frequência observada mostrou valores próximos para as datas de início da estação chuvosa e de cultivo. Ao nível de $20 \%$ de probabilidade, a diferença foi de um dia para o início potencial e de dois dias para o início de sucesso. Para $80 \%$ de probabilidade a diferença foi de três e dois dias para o início potencial e de sucesso, respectivamente. O fim da estação chuvosa e de cultivo ocorreu em 30/09 e 23/10 para as probabilidades de 20 e $80 \%$, respectivamente. A diferença, tanto para a probabilidade de $20 \%$ quanto para de $80 \%$, foi de apenas um dia. $\mathrm{O}$ que indicou que a distribuição dos dados foi aproximadamente normal.

Os comprimentos médios da estação chuvosa e de cultivo (Tabela 3) foram de 209 dias (início potencial) e 202 dias (início de sucesso), ou seja, houve apenas diferença de sete dias. A diferença entre os comprimentos da estação chuvosa e de cultivo também foi baixa para os demais níveis de probabilidade. A $20 \%$ a probabilidade esses períodos ocorreram com 193 e 
182 dias, respectivamente para o início potencial e de sucesso, com onze dias de diferença entre eles. Enquanto que ao nível de $80 \%$ os comprimentos da estação chuvosa foram de 225 (início potencial) e 222 dias (início de sucesso), com diferença de apenas três dias. A diferença entre o comprimento médio pela distribuição de frequência observada e pela função de probabilidade normal foi de apenas um dia, tanto para o início potencial, quanto para o de sucesso. Comparando o comprimento das estações chuvosas e de cultivos pelas duas distribuições observou-se que a $20 \%$ de probabilidade houve diferença de quatro e cinco dias, respectivamente. A $80 \%$ de probabilidade, o comprimento desses períodos, pelas duas distribuições, não teve diferença para o início potencial e diferiu de apenas um dia para o inicio de sucesso. Esses valores mostram, que tanto o início potencial, quanto o início de sucesso apresentaram resultados semelhantes, e assim, pode ser usado qualquer um dos dois na determinação da estação chuvosa e de cultivo.

O conhecimento da data do início da estação chuvosa e de cultivo na agricultura subsidia o planejamento, baseado na disponibilidade de água precipitada, associado às necessidades das plantas, função dos estádios nas quais a demanda de água é maior. Diversas vezes a data do início das chuvas é considerada como a data de semeadura (Arruda e Pinto, 1980). Dessa forma, as culturas agrícolas não sofrerão stress hídrico por causa da menor incidência de períodos secos longos, evitando assim grandes perdas na sua produtividade final.

Resultados do início potencial da estação chuvosa e de cultivo necessitam ser avaliados com atenção, devido ao fato de não considerar uma sequência posterior de dias secos. Portanto, é adequado considerar o início de sucesso, que leva em consideração esse critério. Como exemplo, no ano de 1975, o início potencial da estação chuvosa e de cultivo foi 03/03 e apresentou precipitação total de aproximadamente $35 \mathrm{~mm}$ em dois dias consecutivos. Porém, após 09/03 foi observada a ocorrência de 28 dias secos. O início de sucesso ocorreu em 25/04, sem ocorrência de dias secos posteriores. Durante esse período foi observado um total pluviométrico de $192 \mathrm{~mm}$ ocorridos em 11 dias. Essas informações são úteis em agroclimatologia para garantir condições hídricas favoráveis (precipitação pluvial) ao plantio e o seu subsequente desenvolvimento das culturas agrícolas, tais como, germinação de sementes e estabelecimento de plantas para culturas anuais. Essas definições também são importantes para a cultura de cana-de-açúcar, principal cultivo comercial da região analisada, nas operações de corte, rebrota, tratos culturais e transporte da produção.

A data do início da estação chuvosa e de cultivo para o início potencial e para o início de sucesso foi a mesma em 29 anos (Figura 2). Contudo, em sete anos ocorreram longos períodos secos após a data definida pelo início potencial. Assim, a utilização do início potencial na definição do início da estação chuvosa e de cultivo pode resultar em perdas de produtividade. $\mathrm{O}$ início de sucesso foi o método que mostrou melhores resultados, destacando-se para a definição do início das ocorrências de chuvas. No ano de 2008, o início potencial ocorreu no dia $17 / 03$, tendo aproximadamente $30 \mathrm{~mm}$ de precipitação em dois dias consecutivos. Nos dias seguintes houve sequência de dias com elevada precipitação com $130 \mathrm{~mm}$ em um período de nove dias. Períodos secos também foram observados após o início potencial, porém esses períodos não possuíram sequência longa de dias (menores que 10 dias). Com a ausência de longos períodos secos, o início de sucesso também ocorreu no dia 17/03.

Os modos de variabilidade climática, como a Oscilação Decadal do Pacífico (ODP) e o El Niño - Oscilação Sul (ENOS), também podem influenciar o início da estação chuvosa e de cultivo. Entre 1973 e 1980 houve maior concentração de anos com diferença entre a data do início potencial e o início de sucesso (cinco em sete anos), ou seja, nos 36 anos, ocorreram sete anos com diferença entre o início potencial e o início de sucesso, e desses, cinco anos foram observados entre 1973 e 1980 (Figura 2). O ano de 1993 apresentou diferença significativa entre o início potencial e o início de sucesso. Fato que pode ser explicado pela ocorrência de um evento de El Niño forte, o qual provocou maiores ocorrências de períodos secos longos. Segundo Molion e Bernardo (2002), eventos fortes de El Niño estão associados a ocorrências de secas de moderadas a severas na região. E, como o início de sucesso baseia-se na não ocorrência de períodos secos longos ( $>=10$ dias) nos próximos 30 dias, seus valores diferenciaram significativamente quando comparados ao início potencial.

Em anos de La Niña as ocorrências de chuvas foram antecipadas, ou seja, os eventos de chuvas ocorreram no início de março (início potencial). Porém, essas datas podem ser seguidas por longos períodos secos. No ano de 1985, as chuvas iniciaram em 02/03 e após esse dia houve ocorrência de um

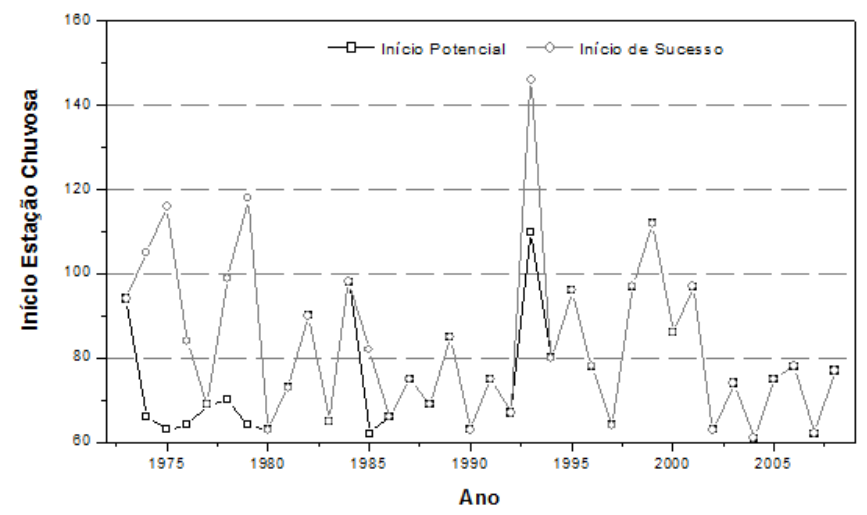

Figura 2 - Comparação entre o início potencial e o início de sucesso da estação chuvosa e de cultivo para a região de Rio Largo - AL. 
período seco de mais de dez dias. O início de sucesso nesse ano ocorreu no dia 24/03. A ocorrência das chuvas foi antecipada em aproximadamente um mês, já que a $80 \%$ de probabilidade o início potencial para os 36 anos foi 01/04. Entre 1974 e 1980 houve ocorrências de eventos de La Niña e El Niño fraco e as ocorrências de chuvas também ocorreram no início de março. Segundo Minuzzi et al. (2007), as ocorrências do fenômeno La Niña proporcionaram maior duração da estação chuvosa na região da Serra da Mantiqueira (São Paulo). Nessa região o início da estação chuvosa ocorreu de forma precoce e o fim foi tardio.

Já com ocorrência de eventos de El Niño forte observou-se que as ocorrências de chuvas foram retardadas. Nos anos de 1982, 1993 e 1998 as ocorrências de chuvas começaram em 30/03, 19/04 e 06/04 para o início potencial e 30/03, 25/05 e 06/04 para o início de sucesso. Comparando essas datas do início potencial e de sucesso com o valor a $80 \%$ de probabilidade para os 36 anos, observou-se que o início potencial e de sucesso para o ano de 1982 ocorreu em datas próximas com apenas dois dias de diferença. O ano de 1998 também apresentou pouca diferença, com apenas cinco dias, tanto para o início potencial, quanto de sucesso. Porém, o ano de 1993 apresentou atraso significativo nas ocorrências de chuvas, sendo a diferença de 18 dias para o início potencial e de 54 dias para o início de sucesso. O evento de El Niño com intensidade forte, que ocorreu nesse ano, provocou a ocorrência de dias secos, em dias nos quais eram esperadas elevadas quantidades de precipitação, e mudou a época de ocorrência de chuvas dessa região. Segundo Minuzzi et al. (2005), eventos El Niño de intensidade forte foram responsáveis pelo início tardio da estação chuvosa nas regiões Norte, Nordeste e Centro de Minas Gerais.

O comprimento da estação chuvosa e de cultivo (Tabela 4) foi menor com ocorrências de eventos de El Niño (1993, 1995, 1997 e 1998). No ano de 1993, que apresentou um evento de El Niño forte, a estação de chuvosa e de cultivo teve 153 dias para o início potencial e 117 para o início de sucesso. Os anos de 1995, 1997 e 1998 apresentaram quantidade total de dias para a estação chuvosa e de cultivo de 179, 193 e 187 dias, tanto para o início potencial, como para o início de sucesso, pois os comprimentos da estação chuvosa e de cultivo para esses anos foram iguais para essas duas metodologias. Nos dois anos seguintes (1998 e 1999) houve ocorrência de um evento de La Niña e a quantidade de dias da estação chuvosa e de cultivo aumentou para 204 e 217 dias, novamente para os dois critérios de início da estação chuvosa e de cultivo utilizados.

\section{CONCLUSÕES}

A estação chuvosa na região de Rio Largo, Alagoas inicia-se, a 80\% de probabilidade, em 07/abril e termina em 24/ outubro, e compreende um período de 221 dias.
Tabela 4 - Comprimento da estação chuvosa e de cultivo para a região de Rio Largo, Alagoas.

\begin{tabular}{|c|c|c|}
\hline Ano & $\begin{array}{c}\text { Comprimento } \\
\text { (Início Potencial) }\end{array}$ & $\begin{array}{l}\text { Comprimento } \\
\text { (Início Sucesso) }\end{array}$ \\
\hline 1973 & 200 & 200 \\
\hline 1974 & 210 & 171 \\
\hline 1975 & 228 & 175 \\
\hline 1976 & 208 & 188 \\
\hline 1977 & 241 & 241 \\
\hline 1978 & 221 & 192 \\
\hline 1979 & 220 & 166 \\
\hline 1980 & 245 & 245 \\
\hline 1981 & 208 & 208 \\
\hline 1982 & 196 & 196 \\
\hline 1983 & 204 & 204 \\
\hline 1984 & 190 & 190 \\
\hline 1985 & 219 & 199 \\
\hline 1986 & 234 & 234 \\
\hline 1987 & 201 & 201 \\
\hline 1988 & 219 & 219 \\
\hline 1989 & 231 & 231 \\
\hline 1990 & 204 & 204 \\
\hline 1991 & 206 & 206 \\
\hline 1992 & 221 & 221 \\
\hline 1993 & 153 & 117 \\
\hline 1994 & 220 & 220 \\
\hline 1995 & 179 & 179 \\
\hline 1996 & 199 & 199 \\
\hline 1997 & 193 & 193 \\
\hline 1998 & 187 & 187 \\
\hline 1999 & 204 & 204 \\
\hline 2000 & 217 & 217 \\
\hline 2001 & 181 & 181 \\
\hline 2002 & 212 & 212 \\
\hline 2003 & 210 & 210 \\
\hline 2004 & 229 & 229 \\
\hline 2005 & 204 & 204 \\
\hline 2006 & 198 & 198 \\
\hline 2007 & 231 & 231 \\
\hline 2008 & 198 & 198 \\
\hline
\end{tabular}

O método direto apresenta resultados satisfatórios na determinação da estação chuvosa e de cultivo (início, fim e comprimento).

Para analisar o início da estação chuvosa e de cultivo, o método do início potencial mostra bons resultados, porém o início 
de sucesso é o recomendado para determinar o início das chuvas.

As ocorrências de eventos de ENOS influenciam o início da estação chuvosa e de cultivo. Ocorrências de El Niño retardaram o início da estação chuvosa e de cultivo, enquanto as ocorrências de La Niña anteciparam o início da estação chuvosa e de cultivo.

\section{AGRADECIMENTOS}

A Fapeal (Fundação de Amparo à Pesquisa do Estado de Alagoas), pelo financiamento deste trabalho através da concessão de bolsa de Mestrado. E as instituições ligadas aos seguintes projetos aprovados: FAPEAL, CT-HIDRO/CNPq504068/03-2, CNPq UNIVERSAL 479143/2007-2, CAPES, CNPq-PIBIC-UFAL.

\section{REFERÊNCIAS BIBLIOGRÁFICAS}

ALLEN, R. G.; PEREIRA, L. S.; RAES, D.; SMITH, M. Crop evapotranspiration: Guidelines for computing crop water requirements. Rome, FAO, Irrigation and Drainage Paper, v. 56, p.300, 1998.

ANDREOLI, R. V.; KAYANO, M. T. ENSO-related rainfall anomalies in South America and associated circulation features during warm and cold pacific decadal oscillation regimes. International Journal of Climatology, v. 25, p. 2017-2030, 2005.

ARRUDA, H. V. de; PINTO, H. S. An Alternative Model for Dry Spell Probability Analysis. Monthly Weather Review, Boston, USA, v. 108, n. 6, p. 823-825, 1980.

AZEVEDO, P. V. de.; MACIEL, G. F. Estação de cultivo e época de semeadura para o algodão herbáceo na região de Sousa-PB. Revista Brasileira de Agrometeorologia, Santa Maria-RS, v. 1, n. 1, p. 81-85, 1993.

CARDIM, A. H. Caracterização da estação de cultivo em Alagoas: análise temporal e espacial. Maceió, 2003. 120 f. Dissertação (Mestrado em Meteorologia) - Instituto de Ciências Atmosféricas, Universidade Federal de Alagoas.

DA SILVA, D. F. Influência dos índices ODP, IME e IOS na variação climática da bacia hidrográfica do rio Mundaú. (Relatório de pesquisa). 2005.

FERREIRA, A. G.; MELlO, N. G. S. Principais Sistemas Atmosféricos Atuantes sobre a Região Nordeste do Brasil e a Influência dos Oceanos Pacífico e Atlântico no Clima da Região. Revista Brasileira de Climatologia, v. 1, p. 15-26, 2005.

KELlER FILHO, T.; ZULLO JUNIOR, J.; LIMA, P. R. S. R. Análise da Transição entre Dias Secos e Chuvosos Usando Cadeias de Markov de Terceira Ordem. Pesquisa Agropecuária Brasileira, v. 41, n. 9, p. 1341-1349, 2006.
MINUZZI, R. B.; SEDIYAMA, G. C.; COSTA, J. M. N.; VIANELLO, R. L. Influência da La Niña na estação chuvosa da região Sudeste do Brasil. Revista Brasileira de Meteorologia, v. 22, p. 347-355, 2007.

MINUZZI, R. B.; SEDIYAMA, G. C.; RIBEIRO, A.; COSTA, J. M. N. El Niño: ocorrência e duração dos veranicos do Estado de Minas Gerais. Revista Brasileira de Engenharia Agrícola e Ambiental, Campina Grande, v. 9, n. 3, p. 364371, 2005.

MOLION, L. C. B. Aquecimento global, El Niños, Manchas Solares, Vulcões e Oscilação Decadal do Pacífico, Climanalise, CPTEC/INPE, agosto, 2005.

MOLION, L. C. B.; BERNARDO, S. O. Uma revisão da dinâmica das chuvas no Nordeste brasileiro. Revista Brasileira de Meteorologia, v.17, p.1-10, 2002.

MORAIS, A. R.; BOTELHO, V. A. V. A.; CARVALHO, L. G.; MUNIZ, J. A.; LAGE, G. Estimativa da precipitação provável em Lavras (MG) através da distribuição Gama. Revista Brasileira de Agrometeorologia, Santa Maria, v.9, n.2, p. 305-310. 2001.

MOUSINHO, F. E. P.; ANDRADE JÚNIOR, A. S.; GONÇALVES, ANDRADE, A. C.; FRIZZONE, J. A. Variabilidade espacial dos percentis 75 da precipitação pluvial anual para o Estado do Piauí. Irriga (Botucatu), Botucatu, v. 11. n. 2. p. 178-187. 2006.

SIVAKUMAR, M. V. K. Predicting rainy season potential from the onset of rains in the Sahelian and Sudanian Climatic zones of West Africa. Agricultural and Forest Meteorology. v.42, p. 295-305. 1988.

SOUZA, J. L.; MOURA FILHO, G.; LYRA, R. F. F; TEODORO, I.; SANTOS, E. A.; SILVA, J. L.; SILVA, P. R. T.; CARDIM, A. H.; AMORIN, E. C. Análise da Precipitação Pluvial e Temperatura do Ar na Região do Tabuleiro Costeiro de Maceió, AL. Período 1972 - 2001. Revista Brasileira de Agrometeorologia, v. 12, n.1, p. 131 - 141, 2004.

STERN, R. D.; DENNETT, M. D.; DALE, I. C. Analysing rainfall measurements to give agronomically useful results. I. Direct Methods. Experimental Agriculture., v. 18, p. 223-236. 1982.

STERN, R. D.; RIJKS, D.; DALE, I.; KNOCK, J. Instat Climatic Guide. P. 325. October, 2005. 